\title{
TV/Series
}

15 | 2019

La Sérialité en question(s)

\section{La construction d'un tissage émotionnel au féminin dans la série Outlander}

\section{Diane Bénédic-Meyer}

\section{OpenEdition}

1 Journals

Édition électronique

URL : http://journals.openedition.org/tvseries/3574

DOI : $10.4000 /$ tvseries.3574

ISSN : 2266-0909

Éditeur

GRIC - Groupe de recherche Identités et Cultures

Référence électronique

Diane Bénédic-Meyer, «La construction d'un tissage émotionnel au féminin dans la série Outlander », TV/Series [En ligne], 15 | 2019, mis en ligne le 16 juillet 2019, consulté le 10 décembre 2020. URL: http://journals.openedition.org/tvseries/3574; DOI : https://doi.org/10.4000/tvseries.3574

Ce document a été généré automatiquement le 10 décembre 2020.

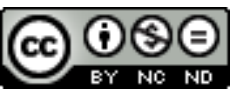

$T V / S e r i e s$ est mis à disposition selon les termes de la licence Creative Commons Attribution - Pas d'Utilisation Commerciale - Pas de Modification 4.0 International. 


\title{
La construction d'un tissage émotionnel au féminin dans la série Outlander
}

\author{
Diane Bénédic-Meyer
}

1 «It's a big old yarn that we're telling here ${ }^{1}$ ", c'est ainsi que Ronald D. Moore, producteur et scénariste en chef de Outlander (Starz, 2014-) définit son adaptation sérielle du roman éponyme à succès de Diana Gabaldon. Le terme yarn fait usuellement référence à la fois au fil du tissage et à un récit d'aventure long et difficile à croire. Le point de départ de l'intrigue est le suivant: Claire Beauchamp, une infirmière anglaise de 1945, est propulsée dans l'Écosse jacobite de 1743. Elle va devoir s'adapter pour survivre et espérer rejoindre son époque et un époux qui l'attend. Elle devra pour ce faire épouser un jeune Highlander du XVIIIe siècle, une rencontre déterminante qui fera tout basculer. Avec quatre saisons diffusées aux États-Unis, la série américanobritannique Outlander obtient un succès populaire et critique notable. Elle a été nominée trois fois aux Golden Globes de 2016 dans les catégories de la meilleure série dramatique, meilleure actrice (Caitriona Balfe), et du meilleur second rôle masculin (Tobias Menzie). En 2017, elle fut la seconde série la plus regardée par les femmes derrière Game of Thrones (HBO, 2011-2019).

2 Outlander représente un cas d'étude pertinent pour explorer la manière dont le format sériel donne à voir la cohérence du personnage. Le tissage émotionnel du personnage se construit sur la durée de la série feuilletonesque où chaque épisode participe à son étirement. Pour reprendre l'image du tissage explicitée par Hélène Breda, le point de vue et les faits et gestes du personnage forment un «fil narratif qui va s'entremêler aux autres personnages au gré des actions de chacun, formant ainsi des motifs narratifs ${ }^{2}$ ».

Le format sériel permet à la fois de rationaliser un contenu riche et parfois décousu et d'intensifier la narration pour mieux donner à voir les facettes du personnage ${ }^{3}$. La linéarité de l'intrigue principale qui repose sur le personnage de Claire n'est qu'apparente. Si le recours au foyer narratif ${ }^{4}$ unique est systématisé, cette unicité est détournée: en définitive, c'est par la fragmentation et le multiple que le tissage 
émotionnel de Claire se construit. La prévalence du regard féminin et la revisite du genre de la science-fiction constituent deux lignes de force de la série Outlander. Tout comme dans Battlestar Galactica (Sci Fi, 2003-2009), Moore engage le spectateur à prendre plaisir à l'immersion répétée dans un monde perdu (l'Écosse jacobite) mais recréé, susceptible de le renvoyer à sa propre réalité et de la questionner. Il existe dans le regard de Claire une authenticité qui renvoie à la sphère de l'intime. Outlander franchit une nouvelle étape pour dire la vérité sur le personnage féminin (sexualité, maternité, traumatismes) par rapport aux séries des années 1990 et $2000^{5}$. Contrairement à Code Quantum (NBC, 1989-1993) ou plus récemment Timeless ${ }^{6}$ (NBC, 2016-2018), la finalité des personnages n'est pas tant de modifier le cours des événements que de vivre avec.

Nous verrons tout d'abord comment la vérité du personnage est donnée à voir par l'établissement d'une tension entre un foyer narratif unique et un perpétuel brouillage des repères. La seconde partie analysera le développement du personnage féminin au sein de la matrice du couple formé par Claire Randall et Jamie Fraser. Enfin nous ferons entrer l'analyse dans le cadre des fan studies pour étudier la réception du personnage de Claire en dehors du texte, en prenant en compte l'action des agents internes (producteurs, scénaristes, acteurs) et externes (communautés de fans) de la série.

\section{Etablir la cohérence du personnage entre brouillage et stabilité}

\section{I.1 Le brouillage des repères}

5 Le brouillage des repères est savamment orchestré tout au long de la série Outlander. L'intrigue initiale repose sur un voyage dans le temps inexpliqué tant pour Claire que pour le spectateur. Confronté à de multiples épreuves, le personnage de Claire s'étoffe. L'adaptation sérielle fonctionne d'autant mieux que Moore a saisi et accentué la démarche de Diana Gabaldon. Il joue avec les codes de genres (historique, romance et science-fiction) et brouille les pistes de l'intrigue pour bousculer les personnages et spectateurs. Le matériau littéraire originel de Gabaldon est inclassable car il n'est pas un roman sentimental au sens strict du terme ${ }^{7}$ et n'a pas de novum qui permette de l'identifier comme une œuvre de science-fiction ${ }^{8}$. Le voyage dans le temps est rapidement relégué au rang de prétexte ${ }^{9}$. La science-fiction s'efface pour se mettre au service de la construction du personnage. En d'autres termes, le cosmographique est mis au service du biographique. Le cadre thématique du voyage dans le temps est le lieu rêvé pour engager un jeu sur le déficit d'information. Dans le premier épisode, la première confrontation entre Claire et les Écossais de 1763 est lourde de tension et d'incompréhension. Aucun des protagonistes en présence ne détient d'informations complètes. Nul n'est capable d'identifier son interlocuteur et encore moins d'appréhender la réalité du saut dans le temps que Claire vient d'effectuer. Elle ne reconnaît pas les lieux qu'elle a certes déjà parcourus mais dans son univers de 1945. Elle porte une robe blanche que les maraudeurs écossais prennent pour la chemise d'une prostituée qui parle comme une anglaise. Ce questionnement quant à l'identité et les motivations de Claire devient un motif récurrent de la série. Les doutes que nourrit le clan McKenzie à propos de Claire la contraignent à épouser Jamie. Le jeu sur le déficit d'information est davantage qu'un ressort de l'intrigue dans le sens où il participe à la 
singularité du personnage de Claire en dressant un mur qui la sépare des autres, à l'exception de Jamie (à qui elle finit par révéler son secret).

6 L'inconnu quant à la suite des événements et au devenir des personnages génère tout autant le brouillage des repères. Il appartient aux scénaristes et au showrunner de maintenir une ouverture des possibles et de proscrire une suite diégétique trop prévisible. L'étape du montage est essentielle car elle nourrit le suspense et donne de la cohérence au personnage. L'usage du flashback et du flashforward (aperçus du récit passé et futur) sont des recours narratifs traditionnels de la série feuilletonesque contemporaine. Outlander ne fait pas exception et use souvent de la prolepse pour susciter la surprise et le questionnement du public. Toutefois, l'épisode pilote donne une portée différente au flashback. L'ouverture de la série présente le récit livré par le personnage de Claire comme une histoire s'étant déjà déroulée et a priori achevée. Or, plus le spectateur avance dans le visionnage, plus il réalise qu'il n'en est rien. La temporalité de la narration demeure incertaine : rien de précis n'est dit sur la situation temporelle de Claire au moment où elle livre son récit. La voix-off provient-elle de Claire à vingt ans, trente ans ou plus? Le seul indice donné est la vision fugitive d'un Écossais présenté de dos et vêtu du kilt traditionnel. On le voit de nuit sous une pluie battante en train d'épier Claire peu de temps avant qu'elle ne débute son voyage dans le temps. Sa chevelure rousse et sa stature sont les signes distinctifs de Jamie que le spectateur ne peut pas reconnaître à ce stade du récit. Le mystère de la présence de Jamie en 1945 pose la question de la fin du récit, qui n'est pas encore écrite ${ }^{10}$.

\section{I.2. Le personnage comme point d'ancrage}

7 Il est aussi difficile d'accéder à la vérité du personnage du fait de ses errements émotionnels. Le réalisme émotionnel ${ }^{11}$ est au cœur de la réussite de Outlander. Les émotions du personnage de fiction sont perçues comme réelles au niveau connotatif par le public qui les reconnaît et les éprouve. Comme l'indique Ron Moore, le spectateur doit pouvoir ressentir le cheminement émotionnel du personnage pour croire à la fable qui lui est contée :

Vous aimez avoir des personnages qui font des choses qu'ils feraient réellement, et lorsque vous regardez la série et que vous en arrivez au point où vous vous demandez "pourquoi donc ferait-elle cela?", «Cela ne lui ressemble pas», ou «c'était une décision stupide- mais pourquoi au nom du ciel...? ?, vous quittez le monde fictionnel, vous n'y faites plus attention, vous n'êtes plus investi émotionnellement dans l'histoire. Donc plus vous êtes fidèles à la réalité de qui ils sont et poursuivez dans cette voie, mieux c'est. ${ }^{12}$

Nous pouvons appliquer au monde sériel la théorie de Brémond sur le récit écrit telle qu'elle fut explicitée par Roland Barthes. La série, au même titre que le récit écrit, doit parvenir à « reconstituer la syntaxe des comportements humains mis en œuvre par le récit, retracer le trajet des choix auxquels, en chaque point de l'histoire, tel personnage est fatalement soumis ${ }^{13}$ ».

9 Ron Moore fait le choix audacieux de se servir du personnage de Claire comme point d'ancrage unique pendant la quasi-totalité de la première saison. Or utiliser un foyer narratif unique dans le format sériel n'est pas sans poser problème car il implique le risque de lasser le spectateur ${ }^{14}$. Dans la série, le personnage de Claire est avant tout une voix. Le premier contact établi entre le spectateur et Claire se fait dès le générique mais de manière indirecte. La voix féminine envoûtante que nous entendons n'appartient 
pas à l'actrice interprète de Claire, Catriona Balfe, mais à la chanteuse Raya Yarbrough $^{15}$. Le thème musical de Outlander est celui d'une vieille comptine écossaise, The Skye Boat Song. Les paroles, issues du poème de Robert Louis Stevenson 'Sing Me a Song of a Lad that Is Gone', narrent la fuite du prince Bonnie Charlie après la défaite de la bataille de Culloden du 16 avril 1746. L'étrange et l'Histoire se mélangent pour former le monde fictionnel.

Le générique de Outlander est construit sur une gradation : la voix de Yarbrough s'élève seule avant d'être progressivement rejointe par plusieurs instruments jusqu'à former une fanfare. La force du générique réside dans l'association d'un élément de stabilité unique que représente la voix féminine et la fragmentation des images qui s'articulent en un mouvement frénétique ${ }^{16}$. La fuite en avant de Claire va de pair avec le tumulte de ses émotions. Nous la suivons via des plans de dos et des gros plans sur ses pieds. De même, nous n'avons qu'une vue partielle des personnages à venir avec une alternance de plans des moitiés inférieures des corps et des visages. L'histoire que nous nous apprêtons à regarder est clairement signalée comme émanant de Claire dans toute sa partialité.

Figure 1 : Claire dans le générique

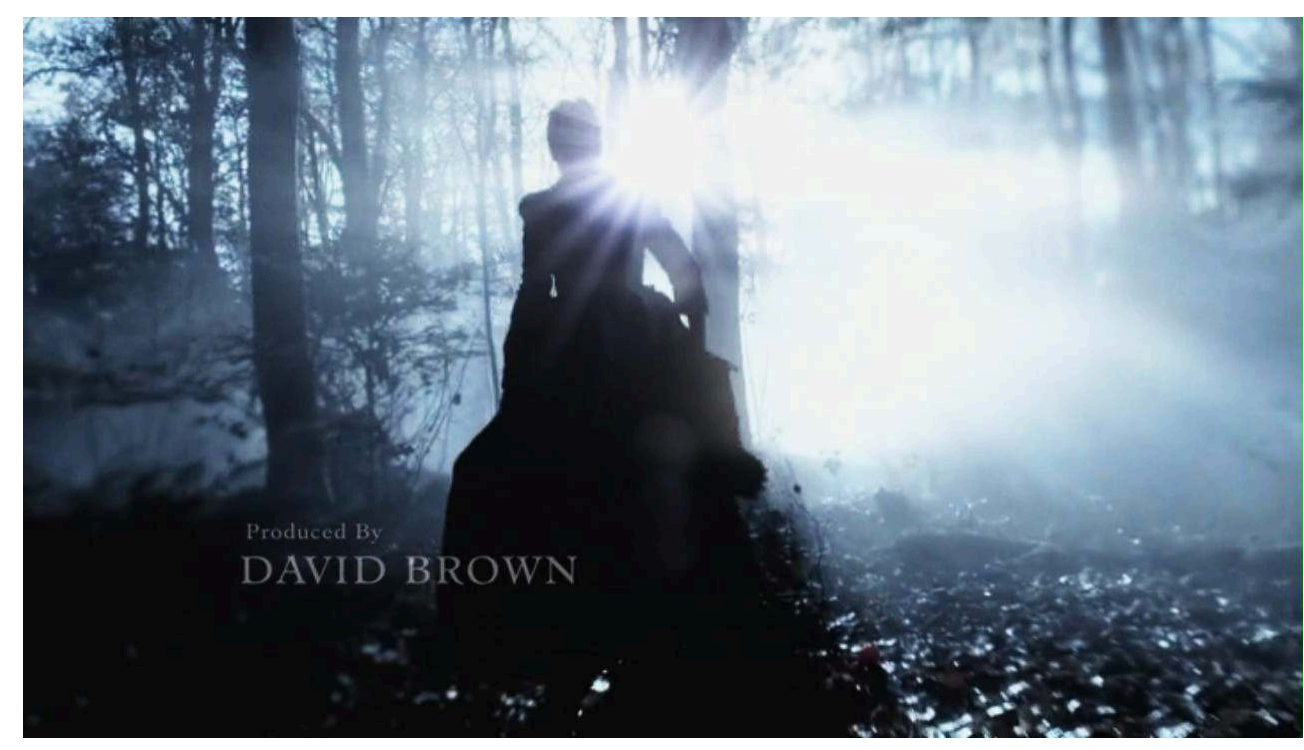


Figure 2 : le visage de Claire dans le générique

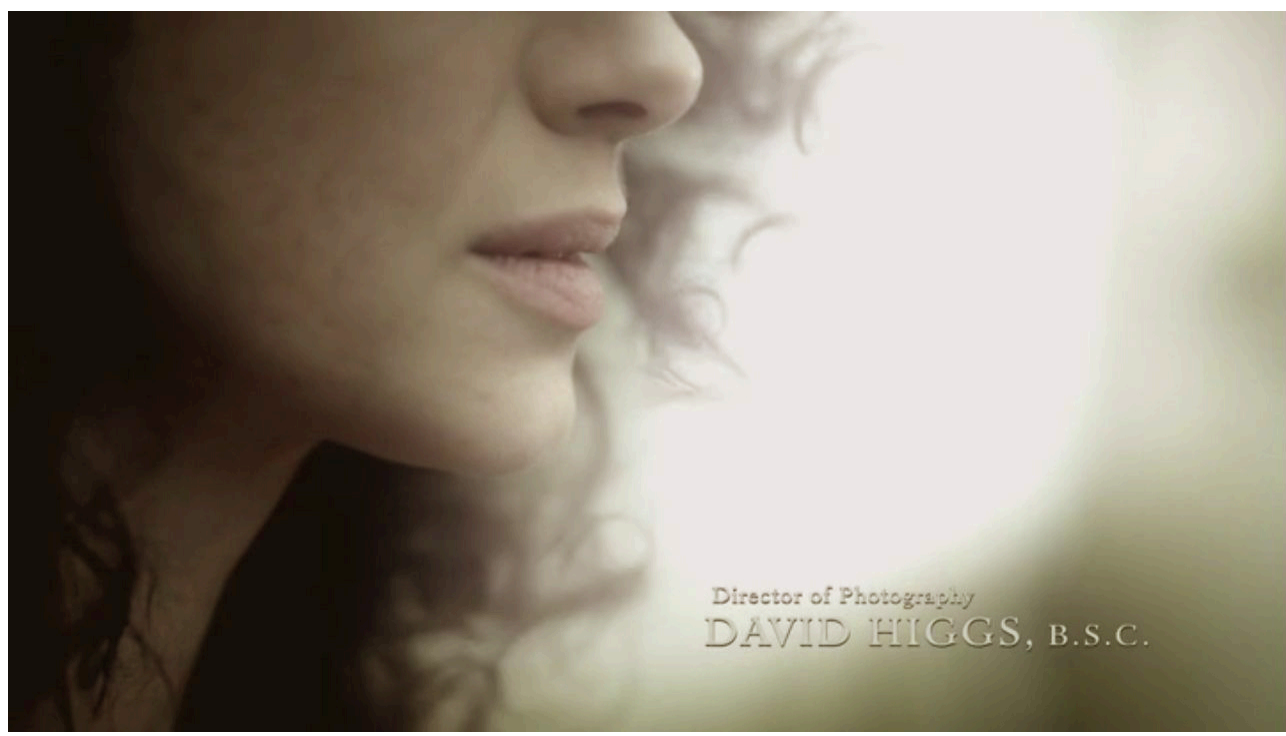

11 Une fois la subjectivité de l'histoire établie, un long prologue donne les clés de la densité du personnage. Comme dans le prologue antique, le créateur de la série veut s'assurer de la bonne compréhension du spectateur. Le prologue de Outlander se distingue par la désaturation des images qui signale que la tranche de vie de Claire se situe avant la diégèse ${ }^{17}$. Elle y est présentée comme une femme indépendante et courageuse, infirmière de la seconde guerre mondiale en pleine action au cours d'une scène d'amputation. La narration en voix-off est utilisée de manière prolongée et répétée afin de demeurer au plus près de ses émotions et ses pensées. La singularité de la situation de Claire fait qu'elle n'a personne à qui se confier à ce moment du récit. Notons que la voix-off au féminin fut longtemps déconsidérée et reléguée au rang de mauvaises pages d'un journal intime. Elle est revenue en force dans les séries américaines depuis Sex and the City ${ }^{18}(\mathrm{HBO}, 1998-2004)^{19}$. Lorsque le spectateur est jugé suffisamment accoutumé à Claire, le recours à la voix-off se raréfie pour laisser davantage de place au couple qu'elle formera avec Jamie ${ }^{20}$.

\section{I.3. Dire l'intimité féminine}

La réussite du tissage émotionnel féminin vient aussi de la capacité à dire l'intimité du personnage féminin. Moore a pris soin de s'entourer d'une équipe mixte "moitié hommes et moitié femmes ${ }^{21}$ ». Cette initiative est malheureusement remarquable quand on sait que les femmes ne représentent que $17 \%$ des metteurs en scène à la télévision en 2015 (selon les chiffres de la Directors Guild of America). Sont recrutés Matthew B. Roberts, Toni Graphia, Ira Behr et Anne Kenney. Les épisodes clés au regard du développement de Claire en tant que femme comme "The Wedding " (S01E07) et «Faith » (S02E07) ont été réservés aux membres féminins de l'équipe.

Outlander adopte le female gaze ${ }^{22}$ afin de retranscrire le regard de Claire. Pour reprendre la définition donnée par Jill Soloway en 2016, le female gaze permet de donner la priorité à l'émotion sur l'action, de capter le regard de ceux qui sont regardés (Jamie dans le cas évoqué ci-dessous) et de savoir retourner le regard (de celui qui est regardé) ${ }^{23}$. La scène de la nuit de noces de Claire et Jamie fonctionne comme une mise en abyme du female gaze en tant qu'objectivation du partenaire masculin. 
Figure 3 : Le female gaze lorsque Claire tourne autour de Jamie pour le regarder

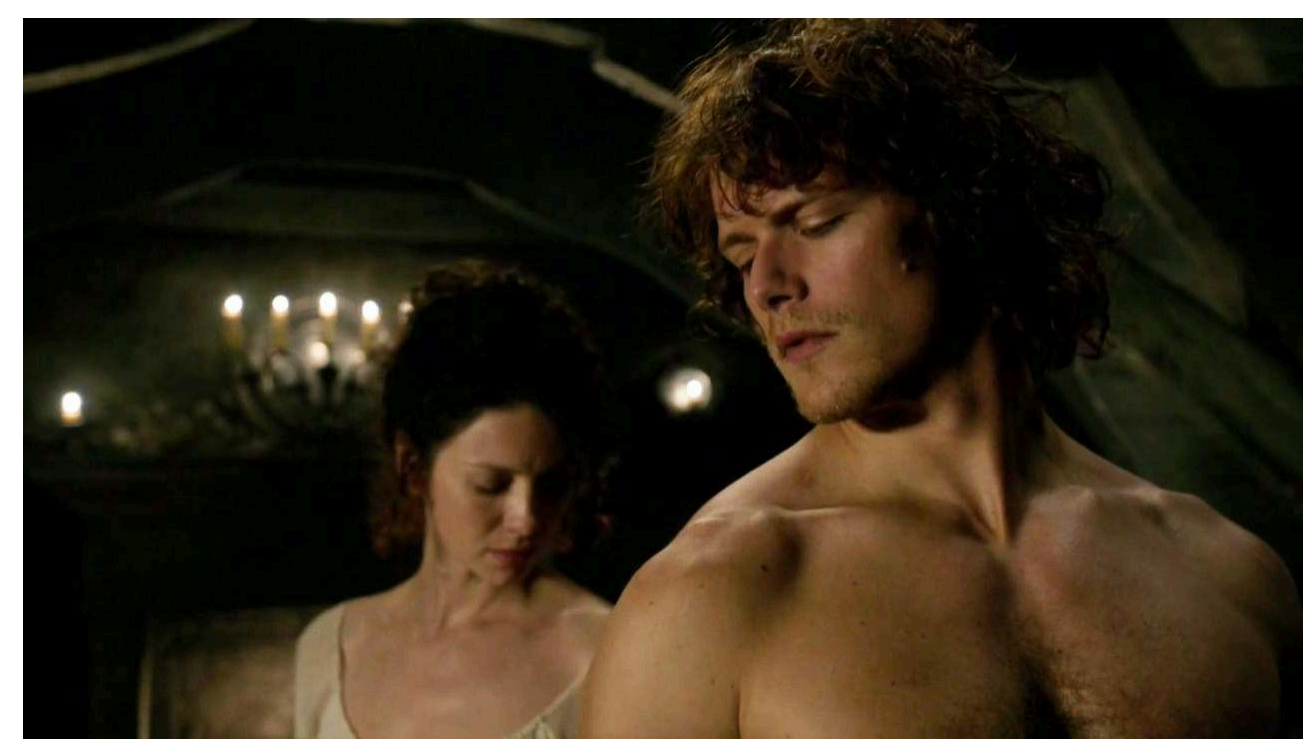

Le traitement du corps et de la sexualité est une donnée importante du personnage de Claire, laquelle apparaît dès le premier épisode comme une femme assumant ses désirs ${ }^{24}$. Elle est montrée de face en train de recevoir du plaisir de son premier mari qui lui est montré de dos. Si la sexualité n'a pas de valeur gratuite puisqu'elle fait avancer l'intrigue, la scène n'en contient pas moins un aspect voyeuriste mais peu susceptible de choquer car elle est destinée à un public féminin ${ }^{25}$. L'épisode "The Wedding" (S01E07) qui traite du mariage entre Claire et Jamie insiste sur la signification émotionnelle de l'acte sexuel. Celui-ci fonctionne sur une gradation en trois temps : la découverte maladroite, la familiarisation avec le plaisir et l'entérinement du lien affectif entre les deux partenaires. Comme l'explique la réalisatrice Anne Foerster, "leur relation sexuelle ou émotionnelle est ancrée dans la manière dont ces trois scènes sexuelles évoluent et elle jette aussi les bases pour ce qui viendra par la suite ${ }^{26}$ $»$.

15 Enfin, le traitement du détail est une autre voie d'accès à l'intimité féminine. La fréquence des gros plans sur les mains jointes de Claire et Jamie à travers les épisodes est significative, tout comme le sont les tranches de vie de femmes qui émaillent le voyage de Claire. Ces espaces constituent à la fois une pause dans le récit et un lieu de vérité. Par exemple, alors que Claire et sa belle-sœur Jenny se lancent sur la trace des soldats anglais qui ont arrêté Jamie, Jenny doit s'arrêter quelques minutes pour soulager ses seins gorgés de lait. La scène est naturelle et le ton demeure pragmatique. D'autres moments féminins apportent une touche de légèreté comme lorsque Claire se mêle aux fileuses d'un village écossais et urine de concert avec les autres femmes pour fixer la teinture sur le tissu. Cependant, le détail n'apparaît jamais aussi pertinent que lorsqu'il amène le spectateur à réfléchir sur sa propre réalité. Le public féminin qui sourit en voyant Claire lutter pour allumer la cuisinière de sa cuisine est ainsi renvoyé au rôle social assigné à la femme de la société américaine des années 1950. 


\section{Le tissage émotionnel féminin au sein de la matrice du couple}

\section{II.1. Le couple comme lieu de développement du personnage}

La construction du tissage émotionnel de Claire se fait au sein de la matrice du couple qu'elle forme avec Jamie. Dans le corpus étudié, le triangle amoureux que Claire forme avec ses deux époux constitue l'intrigue macroscopique. Le dilemme de Claire - qui choisir, Frank ou Jamie? - se manifeste via deux thèmes musicaux. L'un relève du couple formé par Claire et Jamie, l'autre du couple Claire et Frank, comme le précise le compositeur McCreary :

Il n'y a pas de thème de Claire. J'écris la partition du contexte qu'elle vit. Cependant, j'ai tendance à considérer le thème de Claire et Jamie comme son thème à elle. On l'utilise tout au long de la série et je pense que cela contribue à nous guider sur le chemin de leur arc, de leur voyage ${ }^{27}$.

La relation de Claire et Jamie prend rapidement le pas sur le couple de Claire et Frank. Elle est rythmée par trois sommets émotionnels : lorsque Claire choisit de rester avec Jamie dans l'Écosse jacobite (S01E11), quand elle organise l'évasion de Jamie hors des murs de la prison de Wentworth (S01E15) et quand elle sauve Jamie du désespoir (S01E16).

La dynamique narrative est directement impactée par l'évolution du tissage émotionnel du couple lorsque les scénaristes décident de briser le foyer narratif unique. Dans l'épisode The Reckoning (S01E09) où Jamie tire Claire des griffes de Black Jack Randall, tout se passe comme si Jamie avait pris suffisamment d'ampleur dans le champ émotionnel de Claire pour être autorisé à recevoir le flambeau narratif. L'emploi des deux foyers narratifs (celui de Claire et Jamie) est systématisé dans la saison 2.

De plus, le tissage émotionnel du couple se construit par opposition aux éléments extérieurs. Tout comme le foyer narratif unique de Claire représentait un élément de stabilité dans un univers marqué par le danger, le couple devient un lieu de paix et de stabilité. "You are my homeland " ( Tu es ma terre natale »), laisse échapper un Jamie soulagé d'avoir sauvé son épouse (S01E09). Paradoxalement, c'est précisément à cet instant que le titre de la série prend tout son sens. Avant leur rencontre, Claire était l'étrangère en tant qu'Anglaise en territoire écossais et personne venant du futur. Son apparence physique dénuée des stigmates du temps d'une jeune femme du XVIIIe siècle et sa langue sont autant d'attributs de son étrangeté. Jamie quant à lui était l'étranger parce qu'il vivait sous une identité cachée. Le couple qu'ils vont former constitue une unité à part au regard du reste du monde fictionnel.

Le tissage émotionnel du couple se développe entre partenariat et partage impossible. Claire fait entrer Jamie dans une nouvelle dimension sociale puisque de jeune homme il devient homme. En revanche, elle aura fort à faire pour le rallier à ses vues et parvenir à un partenariat susceptible de leur convenir. La sphère privée ne semble guère poser problème. "Fair is fair» («donnant donnant»), énonce Jamie dans la découverte physique de sa partenaire au cours de la nuit de noces. Il en va différemment de la sphère publique comme le montre l'épisode « The Reckoning » (S01E09) où Claire a du mal à accepter le traitement que lui réserve Jamie après qu'elle a mis le clan en danger. Jamie suit les codes du clan et la bat avec une ceinture afin d'asseoir son statut d'homme marié et de réintégrer Claire dans le clan. La scène oscille entre tension et 
légèreté. La musique comique apparaît juste avant la fessée proprement dite. Il s'agit de dédramatiser et de légitimer leurs appréciations différentes de la situation au vu de leur bagage culturel. Claire tiendra sa revanche en pardonnant et en menaçant de châtrer Jamie s'il la violente à nouveau.

Elle dessine vraiment la limite à ne pas franchir. (...) Quand nous avons cette autre scène de sexe intense, je pense que c'est sa manière de dire «regarde, je t'aimerai de toutes les fibres de mon être, mais pas plus que je ne m'aime moi-même, et tant que tu me respecteras, tout se passera bien entre nous ${ }^{28}$.

Caitriona Balfe explique par ces mots que son personnage délimite le cadre du couple. Pour la saison 2, on ne s'étonnera donc pas de voir Claire s'opposer une fois de plus à Jamie. Elle refuse de demeurer dans son hôtel particulier parisien alors qu'elle attend leur premier enfant. Elle est une femme d'action qui dépérit sans activité professionnelle. En dépit de sa grossesse qu'elle devrait dissimuler aux regards du Paris de Louis XIV, elle parvient à se faire accepter à l'Hôtel des Anges où elle s'occupe des malades.

Il est des lieux toutefois où le partage au sein du couple se révèle impossible et où le tissage émotionnel féminin exclue le masculin. L'épisode «Faith» (S02E07) en est un exemple. Celui-ci relate la trajectoire solitaire de Claire partant du désespoir de perdre son enfant mort-né à la réconciliation avec son époux. La réussite de "Faith » repose sur la qualité du scénario, de l'interprétation de Caitriona Balfe et du montage ${ }^{29}$. L'histoire de la souffrance de Claire se déroule principalement dans la cathédrale de Glasgow choisie pour représenter l'Hôpital des Anges. L'épisode d'une heure donne le temps au personnage de Claire d'effectuer son voyage émotionnel et de mettre en scène sa fragilité extrême. Ceci est d'autant plus frappant au regard de son caractère déterminé, de son savoir et de sa résilience remarquable. Les hommes n'ont pas droit de cité dans la chambre où elle gît après avoir perdu son enfant. On la voit seule, le visage filmé en gros plan. La "description intimiste" dont parle Esquenazi est à l'œuvre. Tout se passe comme si on plaçait le personnage féminin «sous une loupe grossissante capable de détailler sentiments et émotions $\mathrm{s}^{30} »$.

Figure 4 : « Faith » (S02E07)

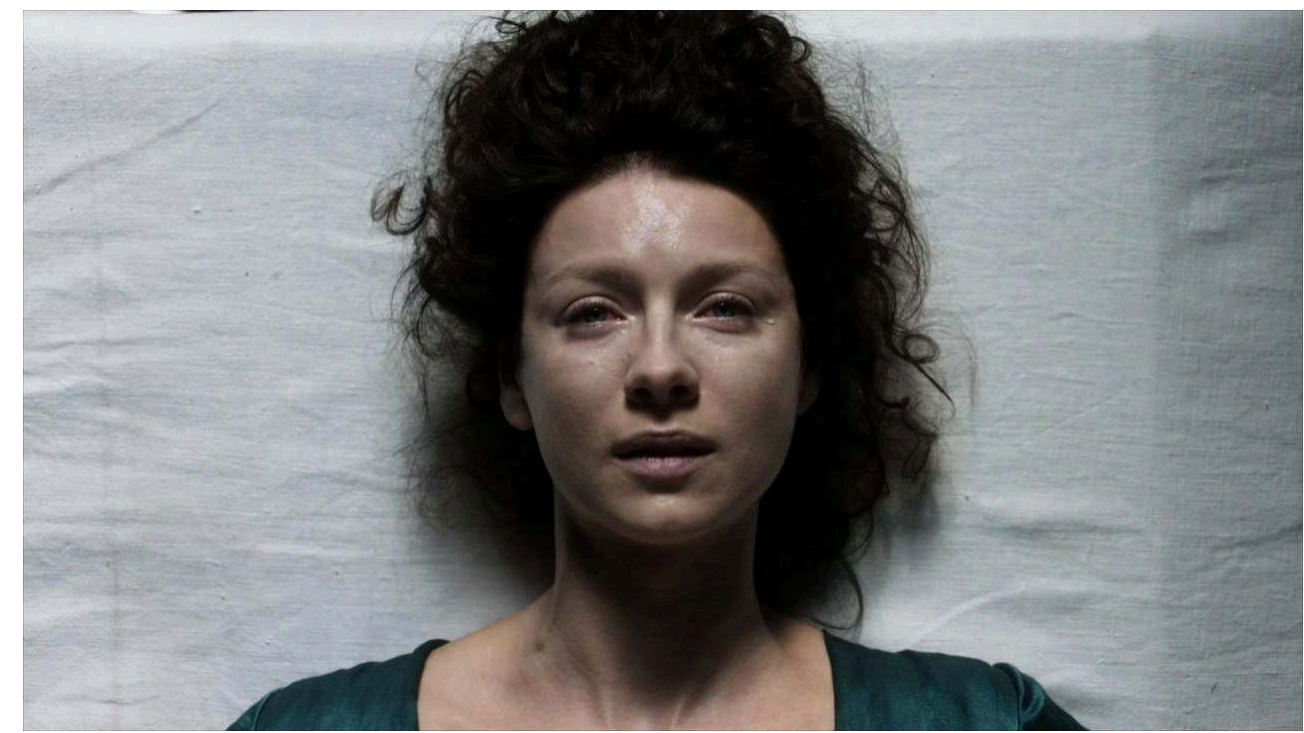



voix $^{31}$ ", dit Ron Moore pour expliquer l'absence de voix-off. Le traumatisme est tel que l'on a l'impression que le personnage s'est dédoublé et son esprit est parti au loin. Le visage défait et blanc se confond avec les draps dans la lumière blafarde. La narration fragmentée traduit la confusion et la dépossession. Après la vision de son désespoir, seule sur son lit, nous voyons Claire bercer son enfant pour la première fois et lui faire ses adieux en la présence de ses deux amies Louise (une mondaine s'épanouissant dans la superficialité chatoyante des salons parisiens) et Mère Hildegarde (administratrice de l'Hôpital). L'ouverture de l'épisode finit de troubler le spectateur en montrant Claire avec une petite fille qui est la sienne mais dont nous apprenons par la suite qu'elle ne peut être sa fille mort-née Faith. L'épisode "Faith» trouvera son pendant dans l'ouverture de la saison 3 avec la naissance de Briana, la seconde fille de Claire. Là encore, le père est exclu (Jamie étant demeuré à son époque et Frank patientant dans le couloir de l'hôpital) et Claire se voit dépossédée de l'expérience de l'accouchement. Elle est endormie de force, une pratique répandue en Nouvelle-Angleterre dans les années 1950 et $1960^{32}$. Le spectateur est bien vite soulagé lorsqu'il se rend compte que l'enfant roux entraperçu auparavant est bien vivant et qu'il s'agit de Briana.

\section{II.2. Une « conversation de l'absence »}

La thématique de l'absence est centrale dans Outlander. Dans la première saison, Frank est physiquement absent bien qu'il envahisse les pensées de Claire régulièrement via le thème musical du couple. Toutefois, un processus narratif différent est à l'œuvre pour expliciter l'évolution conjointe du couple Claire et Jamie. Il s'agit d'un dialogue audible du public seulement qui a ceci de particulier qu'aucun des deux protagonistes n'y prend une part active et n'en a conscience. Nous en distinguons deux modalités. La première a pour objet de densifier un seul personnage tandis que la seconde donne lieu à un entremêlement simultané du tissage émotionnel des deux personnages. La conversation de l'absence du premier type est esquissée dans l'épisode "Je suis Prest " (S02E09). Elle résulte de la mise en parallèle des expériences individuelles de Jamie et Claire alors que tous deux sont engagés dans les préparatifs de guerre contre les Anglais. Le personnage de Claire se développe sur le mode de la superposition et du flashback. Chaque détail du campement écossais la renvoie à son expérience de la seconde guerre mondiale. Il s'agit pour la série de retranscrire le stress posttraumatique dont elle souffre. "Tout le temps, vous essayez de créer un paysage dans sa tête, parce que bien évidemment ce sont toutes les choses dont elle se souvient du passé $^{33}$ ", explique le réalisateur Philip John.

La deuxième modalité de la conversation de l'absence se construit sur la durée. Elle s'étire sur la première moitié de la saison 3. La situation de Claire est la suivante : après une séparation déchirante, elle retourne vers Frank en 1948 pour sauver son enfant à naître. Elle laisse Jamie et ses hommes aller vers une mort certaine à Culloden. 


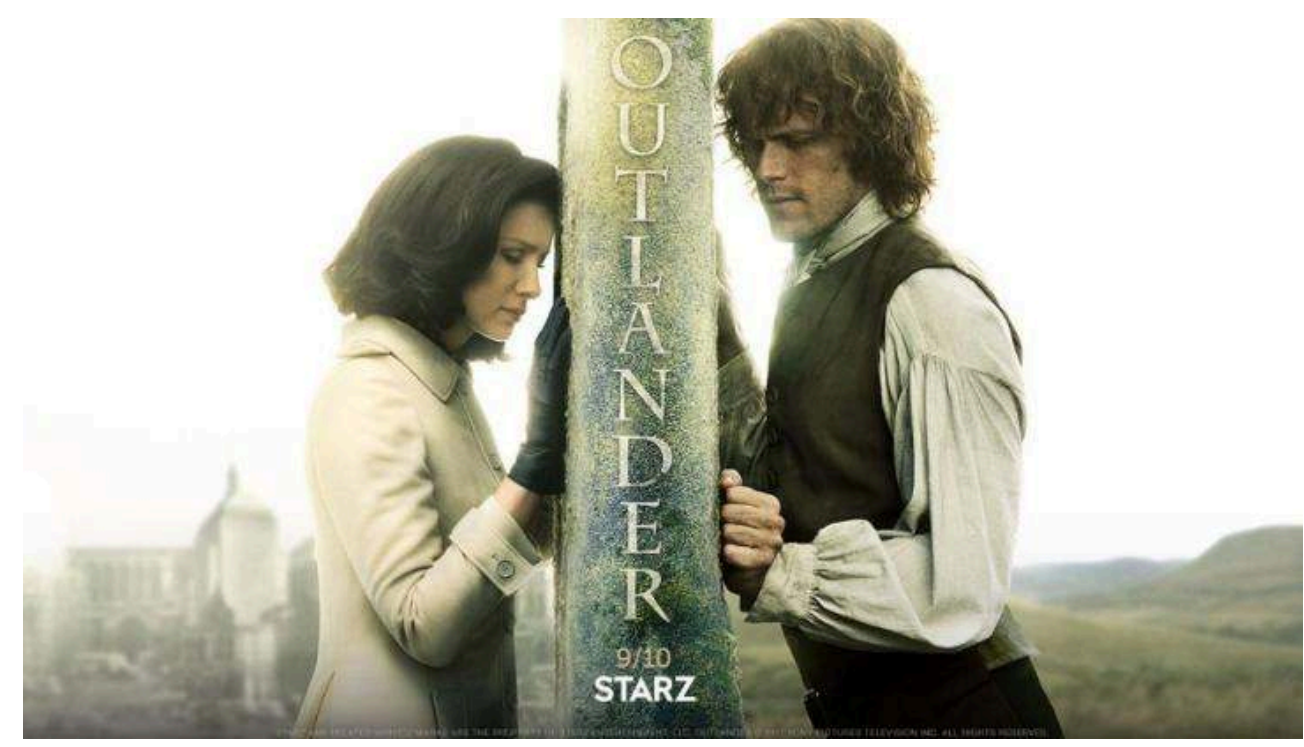

S'engage alors une conversation de l'absence qui transcende le temps et l'espace dont ni Claire ni Jamie n'ont conscience. Les deux foyers narratifs fonctionnent symétriquement, c'est-à-dire en même temps et séparément. Etant persuadée de la mort de Jamie à la bataille de Culloden, Claire refait sa vie avec Frank dans le Boston des années 1950 et 1960. Un jeu d'écho se met en place par complétude rétroactive car le spectateur en sait davantage que le personnage et complète a posteriori l'événement qu'on lui présente. Tout se passe comme si les deux membres du couple entreprenaient un voyage pour se retrouver. Les lignes d'action sont tracées avec un effet miroir: l'histoire de Claire est racontée de l'événement le plus récent au plus ancien tandis que celle de Jamie est narrée dans l'ordre inverse, c'est-à-dire de manière chronologique. La symétrie fonctionne parce que les tranches de vie sélectionnées, si elles appartiennent à des thématiques communes (parentalité, sexualité), ont été vécues à des moments différents. Tous deux par exemple élèvent leur enfant sans pouvoir lui révéler l'identité de leur autre géniteur - Brianna pour Claire (qu'elle a eue de Jamie) et le petit garçon que Jamie a eu avec une aristocrate anglaise. Une analyse plus fine permet de souligner le mimétisme des situations. Deux scènes intimes sont mises en parallèle au sein du S03E02. On y voit Claire et Jamie les yeux fermés tandis qu'ils renouent avec l'acte sexuel. « Tu peux me regarder si tu veux » (you can look at me if you like) de la partenaire de Jamie résonne avec l'injonction de Frank « Ouvre les yeux » (open your eyes) faite à Claire. Ainsi, la conversation de l'absence intensifie la narration et nourrit le tissage émotionnel du personnage.

\section{Réception de la série : la construction du personnage par les fans}

La construction du personnage de série dépend aussi de la réception qu'en ont les spectateurs et les fans. On entend par fan de série un spectateur à fort investissement émotionnel. Le personnage sériel appartient au domaine de la perception par opposition au personnage de roman qui n'a pas d'existence hors du texte. ${ }^{34}$. Le personnage sériel a un interprète qui vit en dehors de la série et auquel on peut 
rattacher la notion de persona (caractéristique des star studies), un type de personnage que le public s'attend à retrouver avec l'interprète qu'il connait. La relation entretenue entre la série et ses fans s'inscrit dans le cadre des relations parasociales. Issu des communication studies des années $1950^{35}$, ce concept désigne à la base une relation à sens unique où une personne (le fan) investit son énergie, son intérêt et son temps émotionnels auprès d'une autre entité (célébrité ou personnage). Jason Mittell adapte ce concept à l'étude du personnage sériel. Il énonce que l'investissement affectif du spectateur pour le personnage sériel est une «composante fondamentale» (key component) de la narration ${ }^{36}$. Ceci est d'autant plus vrai pour Outlander que l'adaptation sérielle a été créée par des fans de la série ${ }^{37}$. De fait, la réception du personnage de Claire est le fruit d'une négociation entre les fans et l'équipe de production de la série. Seront donc analysés ici la culture participative ${ }^{38}$ des fans de Outlander ainsi que les stratégies des producteurs et diffuseurs de la série.

\section{III.1. Nature et action des fans de Outlander}

Si la diffusion d'Outlander s'étend à l'Europe, au Brésil et à quelques pays d'Asie, son public est majoritairement originaire des États-Unis du fait du succès populaire des romans de Diana Gabaldon outre-Atlantique ${ }^{39}$. Ron Moore a d'ailleurs toujours gardé à l'esprit la nécessité de plaire à deux types de publics - ceux qui connaissent l'œuvre de Gabaldon et les autres. Le caractère féminin et intergénérationnel des fans de la série ne fait guère de doute au vu des échanges entre Gabaldon et son lectorat (rencontres, échanges de mails) et du profil des participants aux conventions de la série. La série aborde des thèmes qui les touchent à travers un regard féminin (sexualité, maternité, deuil, difficulté pour les femmes de s'imposer dans une société patriarcale, destinée au long cours du couple). Comme le remarque Chris Parnell, coproducteur à Sony Pictures Television,

« Historiquement, les femmes d'un certain âge se sont senties négligées par l'offre télévisuelle, et nous avons vu un public en demande dont nous savions qu'il voulait une série répondant à ses vœux, qui lui offrait une aventure romantique et mature et pas forcément flatteuse ${ }^{40} \%$.

C'est donc bien le tissage émotionnel au féminin perçu comme authentique par le public qui fait le succès de la série. On comprend mieux pourquoi la plupart des fans avouent n'avoir jamais appartenu à un fandom avant Outlander ${ }^{41}$. La présence de Outlander à la grand-messe du Comic Con de San Diego depuis 2015 marque bel et bien la reconnaissance du public féminin de tout âge par les industries médiatiques, chose impensable il y a dix ans seulement ${ }^{42}$.

Penchons-nous à présent sur la nature des productions des fans d'Outlander. Certes, nombreux sont les sites de fans dédiés à la cosmographie de la série (récapitulatif des épisodes, information sur les personnages et les acteurs) qui dupliquent la fonction d'accompagnement du site de Starz ${ }^{43}$. Néanmoins, les fans savent aller plus loin dans l'appropriation du personnage sériel dans leur usage des réseaux sociaux. Les messages laissés reflètent surtout l'effet émotionnel ressenti par le fan. Ils sont souvent émaillés de GIFs animés ${ }^{44}$ incluant des captures d'écran des expressions faciales des personnages. Les captures d'image de Claire la représentent le plus souvent en couple avec Jamie. Ces GIFs sont avant tout un signe d'appartenance et sont utilisés pour signifier un état d'esprit (à la manière d'un emoji) ou pour dire l'impact émotionnel de l'épisode. La réappropriation des personnages d'Outlander par les fans n'entre pas dans 
le cadre d'une poursuite de la narration ${ }^{45}$ dans ce cas précis. Les contenus transmédia sont le fait d'une petite minorité sous la forme d'une création artistique (dessin, broderie, costume, fan fiction) pour laquelle un talent particulier est requis.

On remarque aussi que les échanges sur les blogs des fans manifestent un va et vient entre le monde fictionnel et la réalité du fan. D'une part, certains fans racontent des événements heureux et moins heureux de leur vie sans entrer dans le détail et de façon apparemment inopinée car sans lien nécessaire avec la thématique de l'épisode. Le deuil est souvent évoqué par des femmes dont les parents sont âgés ou les enfants malades. D'autre part, les échanges sur les réseaux sociaux font état de leur intérêt sans cesse renouvelé pour l'actualité et la vie privée des acteurs. Il arrive que l'hybridité du personnage sériel (qui " procède d'une fusion d'un type particulier entre le personnage et le comédien $\left.{ }^{46} »\right)$ prête à confusion. Les fans sont intrigués et pour certains fascinés par la relation entre les interprètes de Claire et Jamie. La variante du shipping ${ }^{47}$ consiste ici à considérer un couple de fiction comme des amoureux réels. Elle s'opère traditionnellement sur un mode léger et bon enfant entre fans sachant séparer la fiction de la réalité. Toutefois, l'alchimie à l'écran entre les deux acteurs Caitriona Balfe et Sam Heughan est si forte qu'un groupe de hard shippers s'est constitué au sein de la communauté de fans d'Outlander. Ce noyau dur propage la rumeur selon laquelle les acteurs seraient empêchés par leur contrat de se déclarer mais laisseraient des indices sur les réseaux sociaux. La réappropriation du personnage sériel devient pour eux un jeu de piste. Une rumeur nourrissant l'autre, les traces du réel (apparitions publiques, comptes twitter et instagram, et photos volées des acteurs) s'entremêlent aux caractéristiques du personnage sériel. On ne peut que constater l'impuissance de la production et des acteurs à endiguer de telles pratiques malgré des tentatives répétées d'apaisement.

Par ailleurs, les communautés de fan se mobilisent pour nourrir leur relation parasociale ailleurs que sur la toile et provoquer la rencontre avec les acteurs. Elles organisent des conventions qui n'ont que peu à voir avec celles des années 1970 qui avaient pour objet de partager sa passion avec d'autres fans et de consommer des produits exclusifs des franchises de l'univers des comics et de science-fiction. Les participants des conventions des années 2010 achètent leur billet pour s'offrir une expérience émotionnelle avec les artistes et les autres fans présents, le graal étant de pouvoir s'offrir un instant privilégié avec eux en comité réduit. Le succès de la convention est proportionnel à l'intensité de la culture participative du fandom. À titre indicatif, la dernière convention française en date de Outlander (Landcon 3, 2019) a vendu ses billets en une journée. L'impact sur la réception du personnage sériel est toutefois limité car le fan cherche principalement à être reconnu en tant que tel par les acteurs et les autres fans même si les panels sont l'occasion de discuter des personnages avec les acteurs. De plus, les productions sérielles semblent vouloir reprendre la main en cooptant des conventions initiées par les fans.

\section{III.2. Le poids des équipes de Outlander dans la négociation de la réception du personnage}

Les producteurs et diffuseurs de Outlander savent donc pouvoir compter sur la présence d'un fandom très actif. Quelle stratégie de communication déploient-ils pour séduire et fidéliser le public? Comment s'appuient-ils sur le caractère hybride du personnage 
sériel pour peser sur la négociation de la réception? Commençons par la déclaration de John Westphal, vice-président de la programmation à Sony Pictures Television Studios :

«Nous voulons que nos épisodes sonnent juste pour nos fans dévoués. (...) Nous engageons aussi activement le marketing, les médias sociaux, et les équipes publicitaires de Starz et Sony à inclure les fans de toutes les manières possibles ${ }^{48}$. "

La culture participative est au cœur d'une stratégie commerciale pendant et en dehors de la diffusion des différentes saisons. Par exemple, Starz gère l'après-visionnage des épisodes avec la mise en ligne de documents exclusifs comme les corrections manuscrites des scénaristes. Starz encourage l'interactivité avec les réalisateurs, les scénaristes et les acteurs au moyen de forums de questions/réponses éphémères. De façon plus pérenne, un compte twitter (Outlander writers) demeure ouvert aux fans. Les questions pour l'essentiel portent sur l'adaptation sérielle du roman qui tronque certaines de leurs scènes favorites, ce qui ne va pas sans créer des tensions comme le prouve cet échange sur le compte twitter du réalisateur Matthew Roberts.

Fan: "Je pense que cela ne ferait pas de mal d'avoir un consultant chargé de la continuité, lorsque Diana n'est pas disponible.»

M. Roberts : «Il existe un mot pour désigner une personne qui fait toutes ces choses

- les fans » (6 mai 2017) «Chaque cheveu gris a le nom d'un fan d'Outlander dessus.

Un cadeau que l'on continue de me faire. Merci à tous :-) ${ }^{49} »$ (19 mai 2017)

La stratégie commerciale se poursuit entre la diffusion des saisons. Il faut créer l'attente et faire plaisir à un public en état de manque. Ce moment, les fans l'ont euxmêmes baptisé droughtlander (de l'anglais sécheresse). Ils se voient offrir au comptegoutte des extraits des coulisses du tournage ou la mise en ligne de mini-séquences des épisodes de la saison en cours de tournage. La tentative de contrôle d'activité des fans la plus guidée de la part de Starz est très certainement le forum d'écriture ouvert entre les saisons 2 et 3. Les téléspectateurs avaient alors quitté le couple de Claire et Jamie en pleine tragédie mais les fans avertis savaient que la séparation durerait vingt ans comme indiqué dans le matériau d'origine. Starz fournit alors une plateforme d'écriture pour que les fans racontent une histoire d'amour qu'ils avaient vécue et qui elle aussi comportait une longue séparation forcée.

Enfin, on peut mentionner un dernier aspect de la réception du personnage sériel spécifique à la série Outlander. Les acteurs-interprètes de Claire et Jamie encouragent l'activisme des fans en les incitant à soutenir leur action caritative. Ce type de tweets est repris et partagé sur les comptes twitter officiels de la série. L'entrelacement de la vie personnelle du fan, de l'action de l'acteur-interprète et du personnage sériel fonctionne car les valeurs défendues par Caitriona Balfe et Sam Heughan sont en accord avec les personnages qu'ils incarnent. 
Figure 6 : Sweater porté par Caitriona Balfe où l'on peut lire « I'm not the meek and obedient type », une réplique du personnage de Claire.

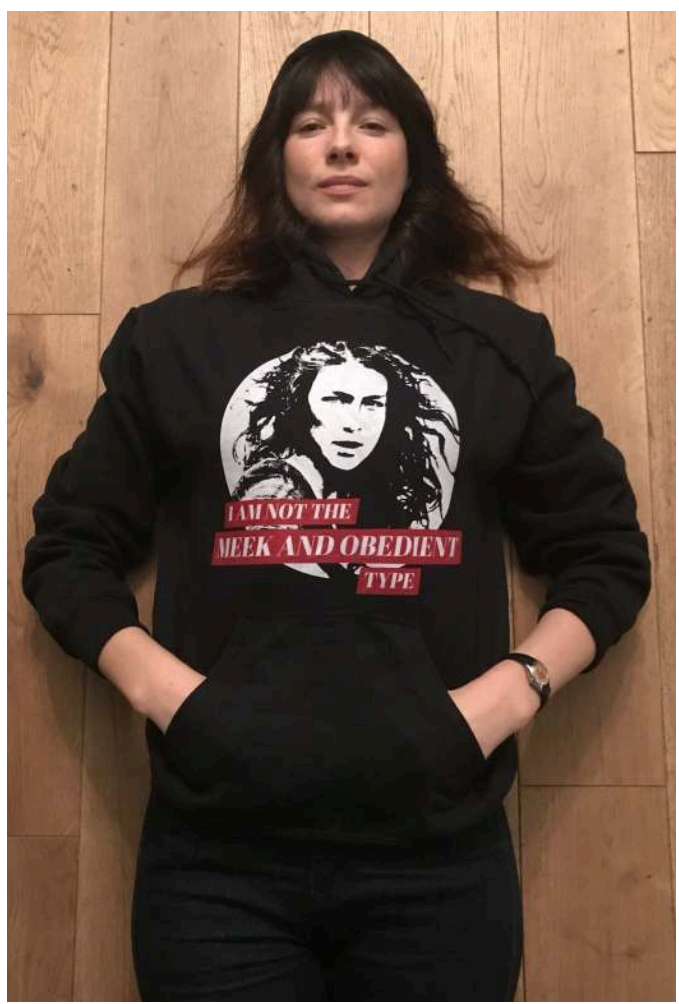

Nul doute que le personnage de Claire se reconnaîtrait dans les causes défendues par Caitriona Balfe. Cette dernière est marraine de World Child Cancer, milite pour l'égalité salariale et contre le harcèlement sexuel (elle soutient le mouvement Time's Up). Elle a aussi soutenu publiquement la demande de retrait du huitième amendement de la constitution irlandaise qui interdit l'avortement.

\section{Conclusion}

Comment le tissage émotionnel au féminin donne-t-il à voir la cohérence du personnage sériel ? La réponse se trouve certainement dans la capacité de la série à construire le réalisme émotionnel du personnage. Le format sériel autorise de nombreux points d'entrée au personnage. Dans Outlander, ce processus passe par la mise en place d'un continuum entre brouillage et stabilité du personnage. Le choix audacieux du foyer narratif unique sur un temps long permet d'asseoir le personnage au sein d'une intrigue mouvante et de la matrice du couple. L'accès à l'intimité du personnage marque le succès de l'entreprise. C'est par le multiple, la fragmentation, le manque et l'absence que s'opère un entrelacement subtil des tissages émotionnels de Claire et Jamie. Le temps sériel permet au tissage narratif de s'étoffer. Il donne naissance dans la troisième saison à un motif narratif inédit - une conversation de l'absence qui s'engage entre les deux êtres séparés et qui fonctionne par la résonnance entre les épisodes.

Par ailleurs, notre incursion dans les fan studies nous a amené à explorer la poursuite du tissage émotionnel au féminin en dehors du temps du visionnage. Le public de Outlander regroupe des femmes de tous âges avec une prépondérance pour la génération du baby- 
boom, un fandom souvent jugé illégitime par les créateurs et diffuseurs de série. La réception du personnage sériel entre dans le cadre d'un paradigme de négociation entre les organismes médiatiques et les fans. Elle se produit dans des espaces de culture participative. L'intensité de l'engagement des fans est l'un des marqueurs de la réussite du tissage émotionnel au féminin dans Outlander, permettant une porosité entre le personnage de Claire, son actrice-interprète et la réalité du fan féminin.

\section{BIBLIOGRAPHIE}

ANG, Ien, Watching Dallas, Londres, New York, Routledge, 1985

BARTHES, Roland, «Introduction à l'analyse structurale des récits », Communications, n 8, 1966, p. $1-27$

BENNETT, Tara, The Making of Outlander: The Series: The Official Guide to Seasons One \& Two, New York, DelaCorte Press, 2016

BREDA, Hélène, Le tissage narratif dans les séries télévisées américaines contemporaines, thèse de doctorat, dir. Gillaume Soulez, Université Paris III, 2015

BOYER, Alain-Michel, Les paralittératures, Paris, Armand Colin, 2008

CHAVON-DEMERSAY, Sabine, « Enquête sur l'étrange nature du héros de série télévisée », Réseaux , n 165, 2011

CONSTANS, Ellen, Parlez-moi d'amour, Limoges, Presses Universitaires de Limoges, 1999

CROWE, Cameron, Conversations with Wilder, New York, Knopf, 1999

CURRIER SWEET, Anne, « "Unreal” Gender Messages in Late 90s Women-Centered Action Dramas », TV/Series, n 1, 2012, mis en ligne le 15 mai 2012, consulté le 21 juin 2017: http:// tvseries.revues.org/1205

DEMASY-QUEFFÉLEC, Lisa, « Du Roman-feuilleton au feuilleton télévisé : mythe et fiction », in MIGOZZI, Jacques (dir.), De l'écrit à l'écran. Actes du cinquième colloque de l'Université de Limoges, Limoges, PULIM, 2000

ESQUENAZI, Jean-Pierre, Les séries télévisées : L'avenir du cinéma ?, Paris, Armand Colin, seconde édition, 2014

FREUND, Katarina, «Becoming a Part of the Storytelling: Fan Vivid Practices and History ", in BOOTH, Paul, A Companion to Media Fandom and Fan Studies, Hoboken, Wiley, 2017

FUSILLO, Massimo, Naissance du roman, Paris, Seuil, 1991

GAY, Roxane, « Outlander Recap: I Believe It's Called Foreplay and We've Been Played Long Enough », New York Magazine: http://www.vulture.com/2014/09/outlander-recap-season-1lashing-engagement.html

GENETTE, Gérard, Figures III, Paris, Seuil, 1972 
HALLEMAN, Caroline, « How Outlander Created a New Breed of Superfan », Town and Country, 3 décembre 2017

HORTON, Donald, WOHL, Richard, « Mass Communication and Para-social Interaction: Observations on Intimacy at a Distance », Psychiatry, n 19, 1956, p. 215-229

JENKINS, Henry, Textual Poachers. Television Fans and Participatory Culture, New York, Routledge, 1992

JENKINS, Henry, Convergence Culture: Where Old and New Media Collide, New York, NYU Press, 2006

JENKINS, Henry, «Panorama historique des études de fans ", Revue française des sciences de l'information et de la communication, $\mathrm{n}^{\circ}$ 7, 2015, mis en ligne le 30 septembre 2015, consulté le 07 mai 2018 : http://journals.openedition.org/rfsic/1645

LAWSON, Richard, « What to Expect from Outlander Season 2 », Vanity Fair, 31 mai 2015

LUTES, Alicia, « Ron Moore and Diana Gabaldon Talk Fandom and Outlander's Many S2 Differences », Nerdist, 8 avril 2016, consulté le 24 avril 2018: https://nerdist.com/ron-moore-anddiana-gabaldon-talk-fandom-and-outlanders-many-s2-differences/

MAROUN, Natalie, Continuité narrative et jeux des possibles dans l'écriture scénaristique des séries télévisuelles, thèse de doctorat, dir. Marc Escola et Charif Majdalani, Université Vincennes-Saint Denis, 2009, p. 38

MILLER, Liz, « Review: Starz's 'Outlander' Needs to Kill the Voice-Over Before The Voice-Over Kills It », Indiewire: http://www.indiewire.com/2014/08/review-starzs-outlander-needs-to-killthe-voice-over-before-the-voice-over-kills-it-23503/

MITTELL, Jason, Complex TV. The Poetics of Contemporary Television Storytelling, New York, NYU Press, 2015

PRUDOM, Laura, « Outlander' Stars Break Down Claire and Jamie's First Fight, That Spanking Scene », Variety, 5 avril 2015: https://variety.com/2015/tv/news/outlander-jamie-clairespanking-fight-reckoning-1201466598/

PRUDOM, Laura, « How Outlander Created Its Most Devastating and Powerful Episode Yet ", Variety, 21 Mai 2016: https://variety.com/2016/tv/news/outlander-recap-season-2-episode-7faith-claire-miscarriage-baby-comte-st-germain-dead-1201780414/

SCOTT, Suzanne, « Revenge of the fanboy: Convergence culture and the politics of incorporation ", thèse de doctorat, dir. Ellen Seiter, University of Southern California, 2010 SOLOWAY, Jill, « Masterclass: The Female Gaze »:http://www.afterellen.com/tv/512369-jillsoloways-keynote-toronto-film-festival-queer-feminist-necessary, consulté le 25 avril 2018 SUVIN, Darko, « On the Poetics of the Science Fiction Genre », College English, Volume 34, $\mathrm{n}^{\circ}$, 1972, p. 372-382

TROUT, Jenny, « Outlander and the Female Gaze: Why Women are Watching », The Huffington Post, 22 septembre 2014: https://www.huffpost.com/entry/outlander-and-the-female_b_5859154

\section{NOTES}

1. Richard Lawson, « What to Expect from Outlander Season 2 », Vanity Fair, 31 mai 2015.

2. Hélène Breda, Le tissage narratif dans les séries télévisées américaines contemporaines, thèse de doctorat, dir. Guillaume Soulez, Université Paris III, 2015. 
3. Le corpus étudié comprend les deux premières saisons de la série ainsi que la première moitié de la saison 3 jusqu'aux retrouvailles du couple de Claire et Jamie après vingt ans de séparation (« A. Malcolm », S03E05).

4. Le foyer narratif est entendu comme «l'origine et le lieu de recoupement » des fils narratifs. Lisa Demasy-Queffélec, « Du Roman-feuilleton au feuilleton télévisé : mythe et fiction », in De l'écrit à l'écran. Actes du cinquième colloque de l'Université de Limoges, éd. Jacques Migozzi, Limoges, PULIM, 2000.

5. Anne Currier Sweet "Unreal" Gender Messages in Late 90s Women-Centered Action Dramas ", TV/Series, $\mathrm{n}^{\circ} 1,2012$, mis en ligne le 15 mai 2012, consulté le 21 juin 2017 : http:// tvseries.revues.org/1205.

6. On peut aussi citer Legends of Tomorrow (The CW, 2016-) et Making History (Fox, 2017).

7. Certes, la narration est faite à la première personne du singulier, la rencontre entre les deux membres du couple n'est pas immédiate et la demoiselle en détresse n'est ni inoffensive ni naïve. Pourtant, Gabaldon inverse avec bonheur le topos de la jeune fille vierge et du héros masculin expérimenté. Pour plus de précisions sur les origines et codes du roman sentimental, on peut se référer aux ouvrages suivants: Alain-Michel Boyer, Les paralittératures, Paris, Armand Colin, 2008 ; Ellen Constans, Parlez-moi d'amour, Limoges, Presses Universitaires de Limoges, 1999 ; Massimo Fusillo, Naissance du roman, Paris, Seuil, 1991.

8. Darko Suvin, «On the Poetics of the Science Fiction Genre », College English, Volume $34, \mathrm{n}^{\circ} 3$, 1972, p. 372-382.

9. La genèse même du livre atteste de la nature accidentelle de l'adoption du genre de la sciencefiction. Comme Gabaldon l'explique sur son blog, elle était dans l'incapacité de faire parler Claire autrement qu'avec l'esprit d'une femme libérée de l'après-guerre au tempérament affirmé. "C'est entièrement de sa faute si le voyage dans le temps fait partie des livres ", dit-elle de Claire. 10. Le romancier ou le showrunner distille à dessein des lacunes dans l'ouverture du récit ou l'épisode pilote afin de "mettre en réserve des possibles narratifs" futurs. Natalie Maroun, Continuité narrative et jeux des possibles dans l'écriture scénaristique des séries télévisuelles, thèse de doctorat, dir. Marc Escola et Charif Majdalani, Université Vincennes-Saint Denis, 2009, p. 38.

11. Ien Ang, Watching Dallas, Londres, New York, Routledge, 1985.

12. "You like to have the characters do things they really would do, and when you're watching a show and you get to a point where you go 'why would she ever do that ?', 'That doesn't seem like her,' or 'that was a dumb choice- why on earth... ?, you check out of the drama, you're no longer paying attention, you're no longer emotionally invested in the story. So the more you can be truthful about who they are and keep them in that lane, the better.» (La traduction de l'anglais vers le français de l'ensemble des citations de cet article sont de mon fait). Alicia Lutes, "Ron Moore and Diana Gabaldon Talk Fandom and Outlander's Many S2 Differences ", Nerdist, 8 avril 2016, consulté le 24 avril 2018: https://nerdist.com/ron-moore-and-diana-gabaldon-talkfandom-and-outlanders-many-s2-differences/

13. Roland Barthes, «Introduction à l'analyse structurale des récits », Communications, $n^{\circ} 8,1966$, p. 1-27.

14. La maîtrise de la voix-off est chose délicate car comme l'énonçait le réalisateur américain Billy Wilder : il faut veiller à ne pas ennuyer tout en complexifiant le narratif visuel.

15. L'artiste avait déjà été choisie pour le thème de Battlestar Galactica.

16. Toutes proportions gardées, le générique de Outlander est comme le contre-point de celui de Games of Thrones. Il dit la désorientation et la fragmentation des souvenirs de Claire tandis que le générique de Games of Thrones s'appuie sur l'exposition de la carte des différents royaumes pour guider le spectateur.

17. «La diégèse est l'univers spatio-temporel désigné par le récit ». Gérard Genette, Figures III, Paris, Seuil, 1972. 
18. On peut notamment penser à The Handmaid's Tale (Hulu, 2017-), série reconnue par la critique qui en fait usage pour dire la frustration du personnage de Natasha alors que Harold Pinter, le scénariste du film (Volker Schlöndorff, 1990) s’y était refusé. La série Insecure (HBO, 2016-) en est un autre exemple.

19. Cameron Crowe, Conversations with Wilder, New York, Knopf, 1999.

20. En dépit de ces précautions, l'usage prolongé de la voix-off a ennuyé voire irrité maints spectateurs comme en attestent les compte-rendu du premier épisode par certains journalistes et fans de la série. Liz Shannon Miller, «Review: Starz's 'Outlander' Needs to Kill the Voice-Over Before The Voice-Over Kills It ", Indiewire, http://www.indiewire.com/2014/08/review-starzsoutlander-needs-to-kill-the-voice-over-before-the-voice-over-kills-it-23503/; Roxane Gay, " Outlander Recap: I Believe It's Called Foreplay and We've Been Played Long Enough ", New York Magazine, $\quad$ http://www.vulture.com/2014/09/outlander-recap-season-1-lashingengagement.html.

21. "I wanted a room that was half men and half women. » Tara Bennett, The Making of Outlander: The Series: The Official Guide to Seasons One \& Two, New York, DelaCorte Press, 2016, p. 9.

22. Le female gaze est une expression de plus en plus galvaudée dans la culture populaire sans pour autant avoir de définition précise. Il est à comprendre par opposition au male gaze (regard masculin). Utilisé pour la première fois par la réalisatrice anglaise Laura Mulvey en 1975, le male gaze est devenu un concept fondateur des études féministes du cinéma. S'appuyant sur les travaux de Freud et Lacan, Mulvey postule que la narration de la figure féminine dans le cinéma hollywoodien est construite à travers le prisme de l'homme hétérosexuel dans le but de satisfaire ses pulsions voyeuristes.

23. Jill Soloway, «Masterclass: The Female Gaze», http://www.afterellen.com/tv/512369-jillsoloways-keynote-toronto-film-festival-queer-feminist-necessary, consulté le 25 avril 2018.

24. La tendance avait déjà débuté mais de manière moins franche avec des personnages féminins dont la sexualité était montrée comme une composante mineure de l'expérience féminine. Citons The Good Wife (CBS, 2009-2016), New Girl (Fox, 2011- ), The Americans (FX, 2013- ) et You're the Worst (FX, 2014-).

25. Jenny Trout, "Outlander and the Female Gaze : Why Women are Watching ", The Huffington Post, 22 septembre 2014 : https://www.huffpost.com/entry/outlander-and-the-female_b_5859154 26. Bennett, p. 83. "So basically their sexual or emotional relationship is anchored in the way those three sex scenes evolve and is laying the groundwork for whatever comes after as well. »

27. Bennett, p. 27. «There is no Claire theme. I score her context. However, I tend to think of the Claire and Jamie theme probably as her theme. It's used all throughout the show and I think it helps guide us on their arc, on their journey. ".

28. "She really draws her boundary line," Balfe noted. "When we have that other intense sex scene, I think that that's her saying, 'Look, I will love you with every fiber of my being, but not more than I love myself, and as long as you respect me, then we're good.» Laura Prudom, "Outlander' Stars Break Down Claire and Jamie's First Fight, That Spanking Scene », Variety, April 5, 2015: https://variety.com/2015/tv/news/outlander-jamie-claire-spanking-fightreckoning-1201466598/

29. Toni Graphia a reçu le prix de l'image de la femme pour le scénario de Faith et l'interprétation de Caitriona Balfe lui a valu une seconde nomination aux Golden Globes.

30. Jean-Pierre Esquenazi, Les séries télévisées : L'avenir du cinéma?, Paris, Armand Colin, seconde édition, 2014, chapitre 9.

31. "You knew what she was thinking, you didn't need to hear her voice. ». Laura Prudom, « How Outlander Created Its Most Devastating and Powerful Episode Yet », Variety, May 21, 2016 : https://variety.com/2016/tv/news/outlander-recap-season-2-episode-7-faith-claire-

miscarriage-baby-comte-st-germain-dead-1201780414/ 
32. Cette scène contient de nombreuses similitudes avec la série Mad Men (AMC, 2007-2015) même si dans cette dernière le female gaze est accentué pour faire ressentir au spectateur la désorientation totale liée à ce type d'accouchement. Dans le making of de l'épisode 5 de la saison 3 de Mad Men, Matt Weiner (créateur et scénariste en chef) n'hésite pas à parler d'une « expérience terrifiante " (terrifying experience).

33. Bennett, p. 201. "All of the time, you're trying to create a landscape in her head, because obviously this is all stuff that she is recalling in the past. »

34. Sabine Chalvon-Demersay, "Enquête sur l'étrange nature du héros de série télévisée ", Réseaux, vol.1165, $\mathrm{n}^{\circ} 1,2011$.

35. Horton, Donald, R. Richard Wohl, «Mass Communication and Para-social Interaction: Observations on Intimacy at a Distance », Psychiatry, n¹9, 1956, p. 215-229.

36. Jason Mittell, Complex TV. The Poetics of Contemporary Television Storytelling, New York, NYU Press, 2015.

37. Ron Moore avoue volontiers que son épouse Terry Dresbach, costumière en chef de la série, est une grande fan de Gabaldon tout comme l'est Maril Davis, productrice-coordinatrice de Outlander.

38. Jenkins identifie deux mouvements au sein d'un paradigme du changement : un mouvement de haut en bas (top down) partant des organisations médiatiques vers les spectateurs consommateurs et un mouvement de bas en haut (bottom up) faisant référence à la culture participative des spectateurs. Sur le sujet, on consultera notamment: Henry Jenkins, Textual Poachers. Television Fans and Participatory Culture, New York, Routledge, 1992 ; Henry Jenkins, Convergence Culture: Where Old and New Media Collide, New York, NYU Press, 2006 ; Henry Jenkins, "Panorama historique des études de fans", Revue française des sciences de l'information et de la communication, $\mathrm{n}^{\circ} 7,2015$, mis en ligne le 30 septembre 2015, consulté le 07 mai 2018 : http:// journals.openedition.org/rfsic/1645

39. La série est diffusée dans les pays suivants : Etats-Unis, Canada, Australie, Europe (RoyaumeUni, Danemark, Finlande, Norvège, Suède, Allemagne, Italie, Pays-Bas), Israel, Japon, Nouvelle Zélande et Brésil. Bien que la série soit tournée principalement en Écosse, elle n'est pas aussi populaire au Royaume-Uni qu'aux États-Unis.

40. "Historically, older women have felt neglected in television offerings, and we saw an underserved audience that we knew wanted a show that delivered a wish-fulfilling, romantic adventure that felt adult and didn't pander ». Caroline Halleman, « How Outlander Created a New Breed of Superfan ", Town and Country, 3 décembre 2017.

41. Hallemann, op.cit.

42. Scott souligne la prise en compte uniquement de fans masculins blancs par l'industrie médiatique. Suzanne Scott, "Revenge of the fanboy: Convergence culture and the politics of incorporation », thèse de doctorat, dir. Ellen Seiter, University of Southern California, 2010.

43. Parmi les plus visités, citons outlandertvnews.com, sass3journey.com, adramofoutlander.com, outlandishobservation.com

44. Un GIF animé est un fichier GIF comprenant plusieurs images qui permettent d'obtenir une animation par affichages successifs en boucle. Il est facile à réaliser et à insérer sur un blog car il ne prend que peu de place. Des sites et blogs proposent même des GIFs de Outlander clé en main Les plus accessibles sont http:www.gyphy.com/outlander et http://outlandergifs.tumblr.com

45. Katarina Freund, «Becoming a Part of the Storytelling: Fan Vivid Practices and History », in A Companion to Media Fandom and Fan Studies, éd. Paul Booth, Hoboken, Wiley, 2017.

46. Chalvon-Demersay, p. 184

47. Le shipping se focalise sur des relations romantiques entre personnages souhaitées par les fans. 
48. Caroline Hallman. «We want our episodes to ring true to our devoted fans (...) We also actively engage the marketing, social media, and publicity teams at Starz and Sony to include fans in as many ways as possible. ».

49. "I think it wouldn't hurt to have a continuity consultant, when Diana isn't available ", compte twitter de Matthew B. Roberts, https://twitter.com/themattbroberts?lang=fr

«They have a word for a person who does all those things-fans :-» (...) « Each gray (or grey) hair has an Outlander fan's name on it. A gift that keeps on giving. Thx everyone :-»

\section{RÉSUMÉS}

Avec quatre saisons diffusées et une cinquième en tournage, la série américano-britannique Outlander (Starz, 2014- ) représente un cas d'étude pertinent pour explorer la manière dont le format sériel donne à voir la cohérence du personnage. Créé par Ron Moore, le showrunner de Battlestar Galactica (Sci-Fi, 2003-2009), Outlander prête une attention toute particulière au réalisme émotionnel de ses personnages au sein d'un monde fictionnel marqué par la science-fiction et l'Histoire. Claire Beauchamp, jeune infirmière de la seconde guerre mondiale, se retrouve mystérieusement propulsée dans le contexte mouvementé de l'Ecosse jacobite. Le tissage émotionnel du personnage féminin se construit sur la durée de la série feuilletonesque où chaque épisode est différent et participe à l'étirement du personnage. Le corpus étudié comprend les deux premières saisons de la série ainsi que la première moitié de la saison 3 jusqu'aux retrouvailles du couple de Claire et Jamie après vingt ans de séparation («A. Malcolm », épisode 305).

Cet article entend démontrer comment l'adaptation sérielle permet à la fois de rationaliser un contenu très riche et parfois décousu et d'intensifier la narration pour mieux donner à voir les facettes du personnage. La série se prête d'autant plus à cette démonstration qu'elle débute comme une adaptation fidèle du matériau littéraire d'origine de Diana Gabaldon pour s'en émanciper peu à peu. L'adaptation sérielle se dote de motifs narratifs spécifiques pour dire l'authenticité du regard féminin de Claire comme le foyer narratif unique ou le female gaze. Il sera aussi intéressant de voir comment le tissage émotionnel de Claire se développe au sein de la matrice du couple notamment par le biais d'une conversation de l'absence. Enfin, la prise en compte du genre féminin du public de la série amènera à explorer la culture participative au féminin à l'heure des réseaux sociaux.

With four seasons already broadcasted and a fifth one forthcoming, the American and British series Outlander (Starz, 2014-) is a relevant case study to explore the way the series format manifests the coherence of the character. Created by Ron Moore, the showrunner of Battlestar Galactica, Outlander is effectively paying attention to the emotional realism of its characters in a fictional world that is marked by science fiction and History. Claire Beauchamp, a young nurse from World War II, mysteriously finds herself thrown into the tense historical realm of Jacobite Scotland. The woman's emotional weaving is being built in the long run in a series where each episode is different and contributes to the character's enrichment. The corpus under study is composed of the first two seasons and the first half of season 3 until the reunion of claire and Jamie Fraser after a twenty-year separation (« A.Malcolm », episode 305).

In this article we intend to demonstrate how the series manages to make visible many aspects of the character by rationalizing a rich and somewhat rambling content and intensifying the 
storytelling at the same time. The series is all the more suitable to such a demonstration since it starts like a faithful adaptation of the original book from Diana Gabaldon and progressively emancipates itself. The series endows itself with specific narrative motifs to convey the authenticity of Claire's female gaze. It will also be interesting to see how Claire's emotional weaving is being developed within the couple's matrix especially through the "conversation of absence". Finally, taking into account the feminine gender of the series' audience will lead to an exploration of the woman's participatory culture in the era of digital social networking.

\section{INDEX}

Mots-clés : tissage émotionnel, féminin, personnage, Outlander, couple, science-fiction, Gabaldon, female gaze, fan studies, réception

Keywords : emotional weaving, women, character, Outlander, couple, science fiction, Gabaldon, female gaze, fan studies, reception

\section{AUTEUR}

\section{DIANE BÉNÉDIC-MEYER}

Docteure en études anglophones, Diane Bénédic-Meyer est spécialiste de l'histoire politique sociale et culturelle des Etats-Unis de la fin du XXe siècle et du début du XXIe. Au cours de ses recherches sur le parti démocrate et la montée du conservatisme, elle a pu mesurer le poids de l'image en politique. Son enseignement à Paris-Diderot puis à l'Université de Strasbourg intègre l'étude de l'image dans la culture des Iles britanniques et des Etats-Unis. Elle a également dirigé la section audio-visuelle du lycée Marc Bloch de Strasbourg.

Diane Bénédic-Meyer est l'auteure d'articles portant sur le pouvoir de l'image en politique (Les années Clinton : la personnalisation excessive de l'exécutif in Robert, F. (Dir.), L'empire de l'Exécutif : la présidence des Etats-Unis de Franklin Roosevelt à George W. Bush (1933-2006), Ellipses, 2008) et sur les guerres culturelles aux États-Unis ( "L'ascension des conservateurs et le traitement de la délinquance aux Etats-Unis : le cas du Violent Crime Control and Law Enforcement Act (1994)», Crime, histoire \& Sociétés, vol. 20, n², 2016 ; Chapman, R. (ed.), Encyclopedia of the Culture Wars, Issues, Voices, and Viewpoints, Armonk, M.E. Sharpe, 2009). 